\title{
Standounkt
}

\section{Die Demokratisierung des Energiesystems am Scheideweg}

\author{
Für die Umsetzung der Energiewende ist eine Demokratisierung \\ und Dezentralisierung der Energieerzeugung notwendig. \\ Doch die deutsche Gesetzgebung torpediert diese wichtige \\ Entwicklung. \\ Von Udo Sieverding und Holger Schneidewindt
}

D ie Energiewende hat zu einer Demokratisierung des Energiesystems geführt. Immer mehr Verbraucher werden von passiven Konsumenten zentral erzeugter Elektrizität und Wärme zu aktiven Marktteilnehmern: Mit Wärmepumpen und solarthermischen Anlagen erzeugen sie Wärme aus alternativen und erneuerbaren Energiequellen und mit Photovoltaik- und Windkraftanlagen produzieren sie grünen Strom. Mini-Blockheizkraftwerke (BHKW) in den Kellern liefern sogar beides. Nachbarn wollen sich zunehmend gegenseitig mit Energie versorgen oder Vermieter ihre Mieter. Und im „Smart Home“ wird der Verbraucher Manager eines eigenen Energienetzwerks, das ihn über ein übergeordnetes Smart Grid mit anderen Akteuren des Energiemarkts verbindet.

\section{Drohkulisse}

Diese Errungenschaften werden durch die aktuell auf nationaler und europäischer Ebene diskutierten Rechtsakte aufs Spiel gesetzt. Statt eines „demokratischen" Energiesystems mit dezentraler Erzeugung, großer Akteursvielfalt und alternativen und erneuerbaren Energien im Zentrum droht ein zentralistischer Ansatz mit alten und neuen Oligopolen, in dem Privathaushalte und Gemeinschaften kaum Beteiligungsmöglichkeiten haben und Erneuerbare Energien nur als Ergänzung geduldet werden.

In Deutschland werden insbesondere das Strommarktgesetz und die diesjäh- rige Novelle des Erneuerbare-EnergienGesetzes über das Schicksal von Prosumern entscheiden. Und in Brüssel baut sich mit dem im Rahmen der Pläne für eine Energieunion angekündigten vierten Binnenmarktpaket, den Beihilferichtlinien und der Überarbeitung der Erneuerbare-Energien-Richtlinie eine gewaltige Drohkulisse auf.

Der zukünftige regulatorische Rahmen für Eigenerzeugung und Eigenverbrauch, insbesondere auch unter Einsatz von Stromspeichern, wird diesen Richtungsstreit mitentscheiden. Dabei muss das ureigene Recht auf Eigenversorgung mit den Folgen der Entsolidarisierung bei der Finanzierung der Energiewende in Einklang gebracht werden.

Mieterstrom-Modelle, also die Versorgung von Mietern durch eine Solaranlage auf dem Dach oder ein BHKW im Keller des Mietgebäudes, bieten in diesem Zusammenhang die Möglichkeit, dass auch Mieter von den positiven Entwicklungen des Energiesystems profitieren. Der deutsche und europäische Gesetzgeber muss Mieterstrom-Modelle daher regulatorisch vereinfachen und fördern, jedenfalls nicht noch weiter erschweren.

\section{Schöne neue digitale Energiewelt?}

Von entscheidender Bedeutung für den Ausgang dieser Debatte ist die zukünftige Rolle von Verbrauchern und Prosumern in der digitalisierten Energiewelt. Sie drohen von den aktuellen politischen Digitalisierungsplänen weg- geschwemmt zu werden. Denn die politischen Entscheider sehen in der Digitalisierung in erster Linie Wachstumschancen für die Wirtschaft und einen Jobmotor für den Arbeitsmarkt, Verbraucher- und Datenschutz haben das Nachsehen. Beschönigende Verweise auf die Energiewende (z. B. „Gesetz zur Digitalisierung der Energiewende") und Verbraucher (EU: „New Deal for energy consumers") sind wenig glaubwürdig und irreführend.

Die Energiewende bietet Verbrauchern als dezentrale Graswurzelbewegung vielfältige interessante und lukrative Beteiligungsmöglichkeiten. Doch der Demokratisierungsprozess ist an einem Scheideweg angelangt. Mit Nachdruck ist davor zu warnen, Verbraucher als Zahlmeister und mit ihrer Wohninfrastruktur, ihren Daten und ihrer Kaufkraft als sogenannte Ermöglicher für Wirtschaftsförderungsprogramme zu Statisten $\mathrm{zu}$ degradieren. Stattdessen muss eine Willkommenskultur für Prosumer regulatorisch abgesichert werden. Mieterstrom-Modelle und Eigenerzeugung/versorgung sind wichtige Instrumente für eine gerechte Verteilung des Energiewende-Nutzens. Verbraucher und Prosumer dürfen nicht mit "nice to have“Argumenten an die Kandare genommen werden, beispielsweise durch eine Zwangsdigitalisierung und -integration ins Smart Grid.

Nur mit beziehungsweise durch starke Verbraucher kann die Energiewende erfolgreich gemeistert werden. Die Erfolgschancen sind umso höher, je mehr Verbraucher auf Augenhöhe mit den anderen Marktteilnehmern agieren und als aktive Prosumer von den Chancen der Energiewende auch profitieren, statt sie nur zu finanzieren.

AUTOREN + KONTAKT

Udo Sieverding leitet den Bereich Energie der Verbraucherzentrale NordrheinWestfalen, Holger Schneidewindt ist dort Referent für Energierecht.

Verbraucherzentrale NRW, Mintropstraße 27, 40215 Düsseldorf. Tel.: +49 211 3809-256, E-Mail: udo.sieverding@verbraucherzentrale.nrw.
Non-Commercial No Derivates License (http://creativecommons.org/licenses/ by-nc-nd/4.o/deed.de), which permits copying and redistributing the material in any medium or format, provided the original work is properly cited, it is not used for commercial purposes and it is not remixed, transformed or built upon. 